\title{
Comparison of the rebuilding effects of different computed tomography scanners and reconstructive settings for the five-line sign in normal interlobular fissures
}

\author{
Anle $\mathrm{Yu}^{1^{*+}} \mathbb{D}$, Qun Li ${ }^{2+}$, Yuefu Zhan ${ }^{3^{*}}$ and Jinlong $\mathrm{He}^{4}$
}

\begin{abstract}
Background: Based on the images generated from two multi-slice computed tomography (CT) scanners, we intended to compare the five-line sign of normal interlobular fissures produced on axial or oblique maximum intensity projection (MIP) reconstructions using different algorithms.

Methods: Two groups of 50 subjects underwent either 16-slice or 256-slice spiral unenhanced chest CT. None of them in either group displayed any abnormality. For each case, maximum intensity projection (MIP) data were used to calculate the axial or oblique projection using four algorithms: standard axial, standard oblique, high-resolution axial, and high-resolution oblique algorithm. The results were then used to reconstruct images of six locations of the lung. The clarity of the five-line sign of the reconstructed MIPs for the interlobular fissures was determined and graded as 1 (unclear), 2 (barely clear), or 3 (clear). Comparisons of the rate and the degree of clarity were performed using nonparametric tests.
\end{abstract}

Results: Data from both the 16-slice and 256-slice CT revealed that the standard oblique algorithm was the best among the four methods for presenting clear images of the five-line sign $(p<0.001)$, whereas the high-resolution axial algorithm was the worst. In addition, the two CT units exhibited no significant differences in the clarity of the five-line sign $(p=0.273)$.

Conclusions: The standard oblique algorithm was the best approach to reveal the five-line sign of normal lung fissures. Both 16-slice and 256-slice CT were effective for reconstructing the sign.

Keywords: Computed tomography (CT), Multi-slice, Five-line signs, Interlobular fissures, Maximum Intensity Projection (MIP), Image processing, Computer-assisted, Image reconstruction

\section{Key points}

- Both 16-slice and 256-slice CT were effective for reconstructing the five-line sign of normal interlobular fissures

- The standard oblique algorithm was the best approach to reveal the five-line sign

\footnotetext{
*Correspondence: yallq2004@163.com; zyfradiology@163.com

${ }^{\dagger}$ Equal contributors

'Department of Radiology, First Affiliated Hospital of Hainan Medical College, 31 Longhua Road, Haikou, Hainan 570102, China

${ }^{3}$ Department of Radiology, Haikou People's Hospital, Haikou, Hainan 570208, China

Full list of author information is available at the end of the article
}

- The MIP-rendered five-line sign has smooth lines and clear gaps and can, therefore, be used as the referencing object for other planar structures in the lung

\section{Background}

Multi-slice spiral computed tomography (CT)-based thin reconstructed images facilitate the clear presentation of various structures and lesions in the lung from any angle [1-3]. Reconstructions based on the maximum intensity projection (MIP) algorithm can reveal the highest level of continuity of linear, high-density vascular structures in the lung. Therefore, this is an excellent method to 
differentiate vascular sections and small pulmonary nodules on a thin-section image $[4,5]$.

Using a 64-slice spiral CT scanner, we previously noticed that normal interlobular fissures presented as multiple parallel shadow lines in MIP-reconstructed images, while the number of shadow lines was equal to the ratio of the thickness of the MIP reconstruction layer to the thickness of the original layer. A five-line sign could be generated if the following parameters were adopted: $1.25-\mathrm{mm}$ layer thickness, $1.25-\mathrm{mm}$ space, and $6.25-\mathrm{mm}$ MIP slab thickness [6]. The five-line sign of interlobular fissures could be used to differentiate the underdeveloped interlobular fissures and the accessory fissures from pulmonary linear fibrosis.

In this study, we examined the effect of two different CT units (16-slice or 256-slice), using 1.0-mm reconstruction layer thicknesses, $1.0-\mathrm{mm}$ space, and also added a high-resolution reconstruction algorithm on the clarity rate and level of the five-line sign.

\section{Methods}

\section{Patients and imaging methods}

This retrospective study was approved by the institutional review board (Haikou People's Hospital and First Affiliated Hospital of Hainan Medical College) with a waiver of informed consent. Candidates were selected based on a consecutive series of apparently normal unenhanced CT examinations performed from December 2012 to April 2013. We included 128 subjects who had no abnormalities on a spiral chest CT scan. Among these, 62 were scanned using 128-row 256-slice CT; 12 of these subjects who had poor-quality images $(n=9)$ or underdeveloped interlobular fissures $(n=3)$ were excluded from the study. The remaining 50 subjects included 35 men and 15 women aged from 18 to 77 years. Sixty-six individuals were scanned using a 16-row 16slice CT unit; 16 of these subjects who had poor-quality images $(n=12)$ or underdeveloped interlobular fissures $(n=4)$ were excluded from the study. The remaining 50 subjects included 28 men and 22 women aged from 18 to 75 years. Here, underdeveloped interlobular fissures means that any segment of the oblique fissures or the major, such as upper, middle or lower one was absent; poor image quality means that there was movement artefact of the bronchi, vessels, fissures and diaphragm on coronal reconstruction in the lungs.

The 256-slice CT unit was a Philips (Philips Healthcare, Amsterdam, Netherlands) Brilliance iCT (Cleveland, $\mathrm{OH}$, USA). Technical parameters included a collimation width of $128 \times 0.625 \mathrm{~mm}$, a rotation speed of $0.75 \mathrm{~s}$, and a pitch of 0.993 . Image reconstruction was performed using two kernels: the standard kernel for the standard algorithm and the lung-enhanced kernel for the high-resolution algorithm. The 16-slice unit was a Siemens (Siemens Healthcare, Erlangen, Germany) Somatom Sensation 16 (Munich, Germany). Technical parameters included a collimation width of $16 \times 0.75$ $\mathrm{mm}$, a rotation speed of $0.5 \mathrm{~s}$, and a pitch of 1.1. The scanner was equipped with two kernels: the B40f kernel for the standard algorithm and the B80f ultra-sharp kernel for the high-resolution algorithm. Both CT units were operated under the following common parameters: voltage $120 \mathrm{kVp}$; tube current $200 \mathrm{mAs}$ (with tube current modulation active on the 16-slice CT unit); 1.0$\mathrm{mm}$ reconstruction slice thickness; $1.0-\mathrm{mm}$ space; 5.0 $\mathrm{mm}$ axial MIP thickness; $6.0-\mathrm{mm}$ oblique MIP thickness; and lung visualisation window (width 1000 Hounsfield units (HU), level - $700 \mathrm{HU})$.

\section{Image analysis}

For multi-planar MIP reconstruction, the 1-mm thinsection-based reconstruction data from the 100 patients were imported into a Brilliance ${ }^{\mathrm{TM}}$ Workspace Portal (V2.6.1.5, Philips Medical Systems, Veenpluis 4-6, 5684 PC Best, Netherlands) to perform multiplanar and MIP reconstruction.

Two orientations were adopted for MIP reconstruction: axial and oblique (oblique coronal). Specifically, reconstruction tangent lines were rotated clockwise or counter clockwise on the sagittal plane until the five-line sign of the pleural segment was clearly displayed on the MIP-reconstructed coronal oblique plane. The angle between the fissure and the tangent line was positive as the tangent line was rotated clockwise from $0^{\circ}$; otherwise, the included angle was negative as the tangent line rotated counter clockwise from $0^{\circ}$; “+” and "-" was marked apart and each of them represented a given direction of the tangent line rotated initially, but did not indicate how small or large the degree of the angle was (Figs. 1 and 2). Bilateral interlobular fissures were divided into upper, middle, and lower zones to be observed. In the right lung the upper segment was above the intersection of the minor with the major fissure, the minor itself as the middle one, and the lower one below the intersection. Since the minor was absent in the left lung, the major fissure of the left lung was divided into the upper, middle, and lower segments for imaging observation. This was also applied to the right lung when the minor fissure was undeveloped or absent.

Each sample was subject to calculation using the standard axial algorithm, the standard oblique algorithm, the high-resolution axial algorithm, and the highresolution oblique algorithm to reconstruct images at six locations in the lung: upper left, upper right, middle left, middle right, lower left, and lower right. Two physicians, Y.Z. and A.Y., with 5 years and 20 years of diagnostic imaging experience, respectively, worked in consensus to 


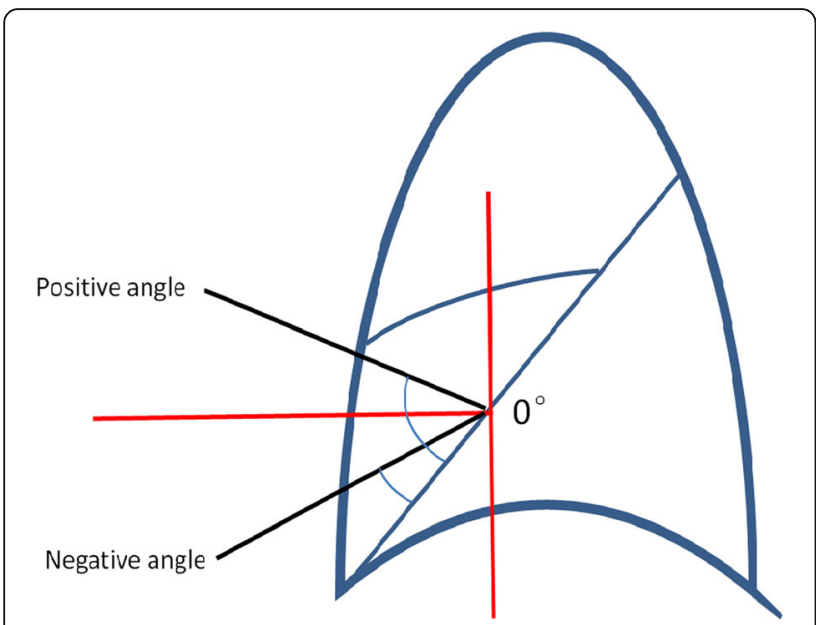

Fig. 1 Sagittal diagram of oblique maximum intensity projection rebuilding to observe the five-line sign. The angle between the fissure and tangent line is positive when the tangent line is rotated clockwise from $0^{\circ}$; conversely, the angle is negative. The long oblique line represents the major fissure, and the short one represents the minor fissure

formulate an image clarity standard for the five-line sign of interlobular fissures in the MIP-reconstructed images.

Based on this standard, the axial and oblique rendering outcomes of the 100 subjects who had normal interlobular fissures were assessed. The clarity levels of the fiveline sign were graded as 1 (unclear, when single lines of interlobular fissures were visible in the original thinsection images, but the MIP-reconstructed image failed to reveal multiple lines and instead displayed band shadows with either a clear or an unclear boundary), 2 (barely clear, when the five lines were distinguishable but with low density, inadequate contrast and blurring gaps), or 3 (clear, when the five lines presented with distinct boundaries) (Figs. 2 and 3). For each controversial case, a third-party expert (Z.H.) with 5 years of experience in diagnostic imaging was consulted until a consensus was reached.

\section{Statistical analysis}

Data analysis was performed using SPSS 16.0 software (Chicago, USA). The Kruskal-Wallis test was used to compare the clarity levels of the five-line sign and the angles between the fissures and tangent lines derived from the two CT units and the four calculation methods. The Kruskal-Wallis test and a subsequent MannWhitney $U$ test were used for multiple comparison analysis of the clarity levels. The $\mathrm{X}$-square test was used to compare the rate of assignment to clarity level 3 and combined $(2+3)$ clarity levels for the four calculation methods and the two CT units as well as the clarity rates of the five-line sign among six locations on axial or oblique positions. The significance level of the statistical analyses was set as $\alpha=0.050$. Inter-observer agreement for clarity levels of the five-line sign from different CT scanners and different algorithms was estimated by using weighted $\kappa$ statistics [7].

\section{Results}

The following settings were used for both CT units: 1.0$\mathrm{mm}$ reconstruction thickness, $1.0-\mathrm{mm}$ space and 5.0 -


Fig. 2 Images from 256-slice spiral computed tomography (CT). a-c Standard algorithm-generated images. Width of the lung window, 1000 Hounsfield units (HU); window level, $-700 \mathrm{HU}$. a At the workstation three-dimensional interface, the left image is an axial maximum intensity projection (MIP) reconstruction with 5-mm slab thickness showing the lower right oblique fissure. The interlobular fissure manifested a bandlike pattern and had high density and a clear boundary. No five-line sign was revealed (white arrow). The basic point (small green cross) was set at the middle point of the lower right oblique fissure, coronal (right upper image), and sagittal plane (right lower image) corresponding to the middle point of the axial lower oblique fissures. $\mathbf{b}$ In the sagittal position (right lower image), which is the same one as shown in $\mathbf{a}$, and the workspace interface, the reconstructed tangent lines were rotated counter clockwise to a given angle (black arrow) to allow formation of an oblique coronal plane (left image). For the interlobular fissure of this oblique plane, a five-line sign was clearly revealed (white arrow) with a MIP slab thickness of 6 mm. c On the sagittal position, which is the same one as the right lower image shown in $\mathbf{b}$, the angle between the tangent lines and the oblique fissure (arrow) is $31^{\circ}$; the angle between the horizontal line and the oblique fissure (arrowhead) is 53; and the angle between the tangent lines and the horizontal line (arrow with a fish tail) is $22^{\circ}$. Notice that the minor fissure is imaged as an arc line-like pattern on the sagittal plane 


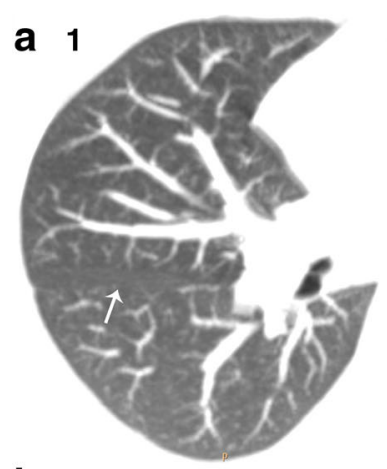

b 1

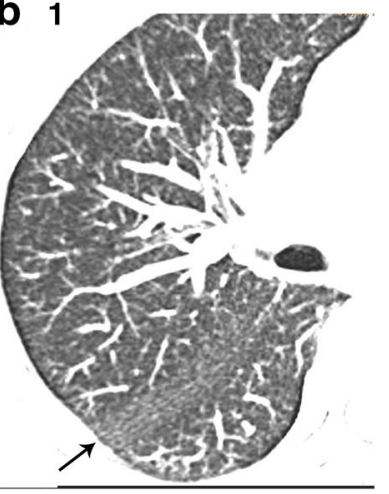

2

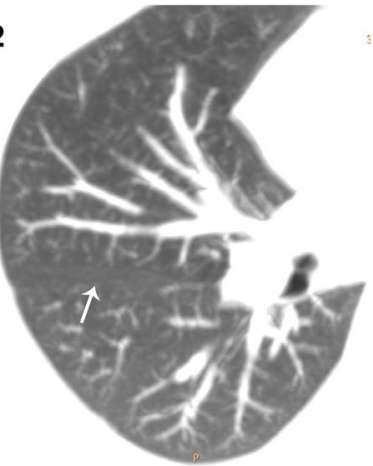

2

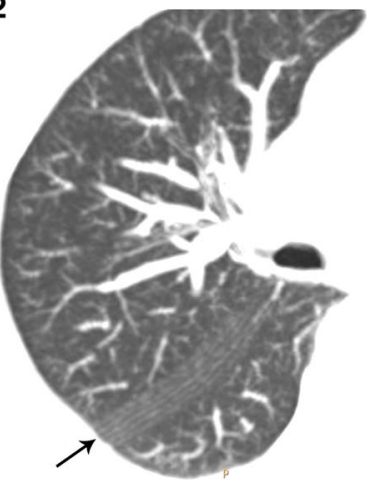

3

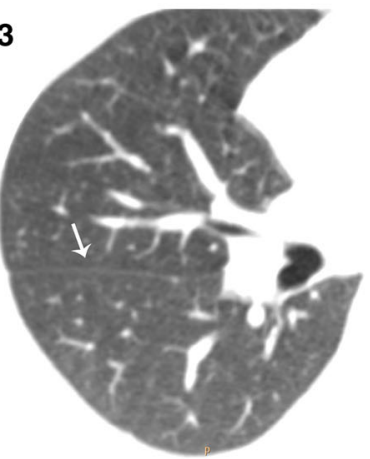

Fig. 3 a Images from 256-slice spiral computed tomography (CT). a1-3 Standard-algorithm-generated images obtained from the same individual. Width of the lung window, 1000 Hounsfield units (HU); window level, - $700 \mathrm{HU}$. a1 Axial maximum intensity projection (MIP)-rendering with a 5-mm slab thickness showing the lower segment of the right major fissures as a low-density band shadow with a blurred edge. No five-line sign was visible (white arrow). a2 MIP reconstruction at the same location as a1 except the scan was from an oblique coronal position. The five-line sign of the lower segment of the right major fissures was still unclear (white arrow). a3 Axial 1.0-mm thin-section image of the same location as in a1 and a2. The lower segment of the right major fissure is manifested as a low-density line with a blurred edge (white arrow). $\mathbf{b}$ Images from 16-slice spiral CT. b1 and b2 Transverse images from the same individual; width of the lung window, $1000 \mathrm{HU}$; window level: - $700 \mathrm{HU}$. b1 Axial MIP reconstruction with 5-mm slab thickness and a high-resolution algorithm. The five-line sign of the upper segment of the right major fissure was barely clear, and some lines were visible (black arrow). b2 Same location as in b1; axial MIP reconstruction with 5-mm slab thickness and the standard axial algorithm. The five-line sign of the upper segment of the right major fissure was clearly presented (black arrow)

mm MIP slab thickness. Correspondingly, both units generated MIP reconstruction images at the axial position that revealed parallel five-line shadow lines for the normal pleura. In other words, the number of shadow lines was equal to the ratio of the thickness of the MIPlayer to the thickness of the original layer (Fig. 3). However, when the images derived from the two units were reconstructed by oblique MIP, the workspace added $1 \mathrm{~mm}$ by itself to reach a thickness of $6 \mathrm{~mm}$ before the five-line shadow lines could be displayed. As the MIP thickness was increased, the line number doubled, resulting in a gradual reduction in the density. Consequently, the surrounding structures blended into the shadow lines, and the external lines gradually disappeared (Fig. 4).

Using 16-slice and 256-slice CT, the standard oblique algorithm presented the highest degree of clarity for the five-line sign $(p<0.001)$, whereas the high-resolution axial algorithm presented the lowest clarity $(p<0.001)$ among the four methods. There were no significant differences in the clarity of the five-line sign using the two
CT devices when the same calculation method was used (Table 2). A comparison of the clarity of the five-line sign obtained from the two CT scanners using the four calculation methods is presented in Table 1.

When the standard oblique algorithm was used, the rates of assignment to clarity level 3 from six locations were $70.3 \%$ for the 16 -slice CT and $74.3 \%$ for the 256 slice $\mathrm{CT}$, which were not significantly different $\left(x^{2}=\right.$ $1.199, p=0.273)$. In the lower left zone, however, 256slice CT yielded significantly superior results to 16 -slice CT (60\% versus $\left.34 \% ; x^{2}=6.784, p=0.009\right)$. If data from level 2 (barely clear) were included, the total clarity rates of the six locations increased to $100 \%$ and $97.3 \%$ for 16 slice CT and 256-slice CT, respectively.

When the high-resolution oblique algorithm was used, 256-slice CT exhibited a higher rate of assignment to clarity level 3 than did 16 -slice CT (48\% versus $14 \%, x^{2}$ $=13.551, p<0.001)$ in the lower right lung area. In addition, there were no significant differences between the two CT scanners in the clarity of the five-line sign at 


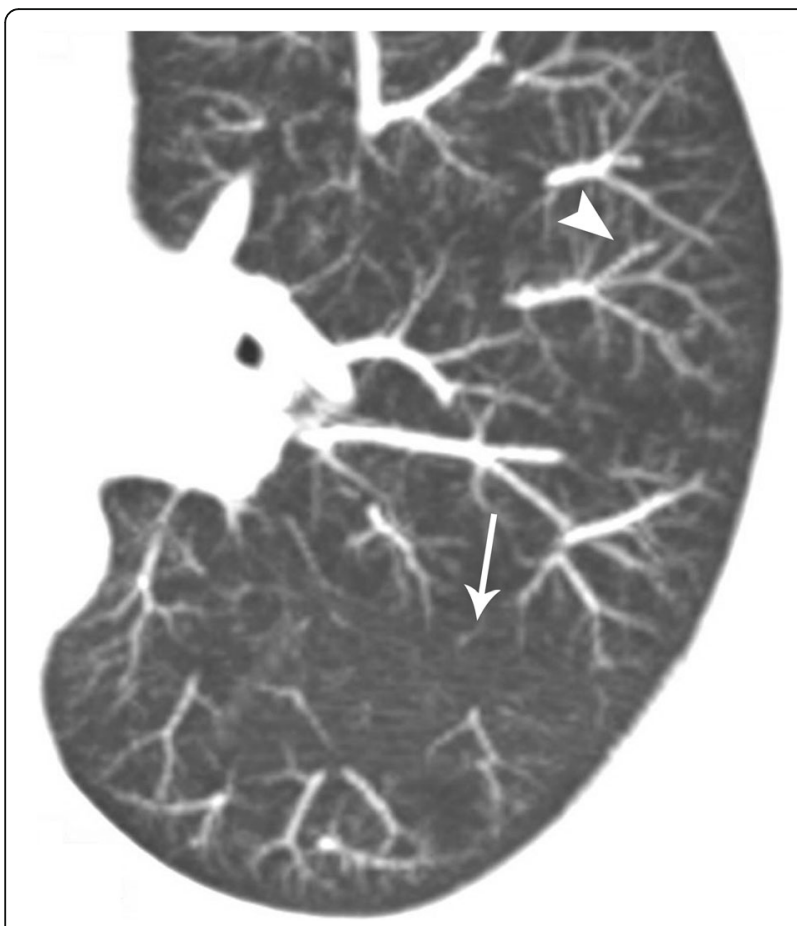

Fig. 4 Images from 16-slice spiral computed tomography (CT). The standard algorithm generated transverse images: width of the lung window, 1000 Hounsfield units (HU); window level, $-700 \mathrm{HU}$. The 12-mm-thick axial maximum intensity projection-rendered image of the upper segment of the left major fissure shows decreased density of multiple shadow lines and the surrounding structures overlapping with multiple shadow lines, with most of the shadow lines being no longer visible (white arrow). Segmented small blood vessels are visible (white arrowhead)

Table 1 Comparison of the clarity levels of the five-line sign derived from different algorithms using 16-slice and 256-slice CT

\begin{tabular}{llll}
\hline Method & Number of sites & \multicolumn{1}{l}{$M\left(P_{25}, P_{75}\right)$} \\
\cline { 3 - 4 } & & 16 -slice & 256-slice \\
\hline HRA & 300 & $1.00(1.00,1.00)^{\mathrm{a}, \mathrm{g}, \mathrm{i}}$ & $1.00(1.00,1.00)^{\mathrm{d}, \mathrm{h}, \mathrm{j}}$ \\
HROA & 300 & $2.00(1.00,2.00)^{\mathrm{b}}$ & $2.00(1.00,2.00)^{\mathrm{e}}$ \\
SAA & 300 & $2.00(1.00,2.00)^{\mathrm{c}}$ & $2.00(1.00,3.00)^{f}$ \\
SOA & 300 & $3.00(2.00,3.00)$ & $3.00(2.00,3.00)$ \\
Sum & 1200 & $2.00(1.00,3.00)$ & $2.00(1.00,3.00)$ \\
H & & 548.621 & 531.787 \\
$p$ & & $<0.001$ & $<0.001$ \\
\hline
\end{tabular}

$C T$ computed tomography, HRA high resolution axial algorithm, HROA high resolution oblique algorithm, SAA standard axial algorithm, $S O A$ standard oblique algorithm. Comparison of HRA, HRO, and SAA with SOA: the $U$ values of ${ }^{2} 2541.50$,

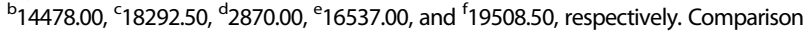
of HRA and SAA: the $U$ values of ${ }^{9} 25832.00$ and ${ }^{\mathrm{h}} 25141.50$, respectively. Comparison of HRA and HRO: the $U$ values of '20910.00 and ${ }^{j} 21283.00$, respectively. The $p$ value was $<0.001$ for all statistical analyses

$M\left(P_{25}, P_{75}\right)$ represents median (25th percentile, 75th percentile) $\mathrm{H}$ in the stub represents the statistic value obtained from formula KruskalWallis test. It is equivalent to the chi square value obtained by chi square test any location when calculated using the same methods. The clarity rates of the five-line sign derived from the different methods and the two CT devices are summarised in Table 2.

Table 3 shows that the five-line signs displayed directly on the axial MIP images in most of the upper segments of the right major fissures and of the minor fissures; however, the oblique lines had to be adjusted to show the five-line sign in the remaining segments of the bilateral fissures.

Table 4 shows that there were no significant differences between the two CT scanners in the angles of the same location between the fissures and the tangent lines, but there were significant differences in the angles among different locations using the same CT scanner.

Inter-observer agreement for the clarity of the five-line sign for the high resolution axial algorithm, the highresolution oblique algorithm, the standard axial algorithm, and the standard oblique algorithm was represented by $\kappa$ values of $0.142,0.559,0.679$, and 0.922 for the 16-slice CT scanner and 0.125, 0.736, 0.617, and 0.827 for the 256-slice CT scanner, respectively. Thus, there was almost perfect agreement for the standard oblique algorithm for both CT scanners.

\section{Discussion}

In the current work, the results obtained from this investigation indicated that the phenomenon of the five-line sign, which we identified previously using a 64-slice CT scanner [6], was present when using different types of CT scanners and under different reconstruction settings.

The phenomenon of the five-line sign or multi-line reflections, which appear within axial MIP reconstructions of interlobular fissures, may be caused by partial volume averaging in the lung $[6,8,9]$ because the signs appear to be the beaded vessels exhibited by MIP-reconstructed pulmonary microvasculature, which was first proposed by Napel [8]. Evidence indicated that the beaded vessels and the multiple shadow lines of interlobular pleura belong to the same type of artificial shadows [6].

Of note, the $1.0-\mathrm{mm}$ thin-slice is commonly used for three-dimensional reconstruction of lung microstructures and nodules [10-12]. We determined that the $1.0-\mathrm{mm}$ thickness is the best for MIP-reconstruction of interlobular fissures, and that too thick or too thin slice thickness is not advisable [6]. In addition, the reconstruction space should be equal to the slice thickness. When the space is larger or smaller than the thickness, overlapping of multiple shadow lines or wider or narrower gaps between the lines can occur. Here, we used 1.0-mm thickness data derived from two CT scanners for oblique MIP reconstructions. In the Philips Brilliance ${ }^{\mathrm{Tm}}$ Workspace Portal, the MIP reconstruction thickness must be increased to 6.0 
Table 2 Comparison of the clarity level of the five-line sign derived from 16-slice and 256-slice CT

\begin{tabular}{|c|c|c|c|c|c|c|c|}
\hline \multirow[t]{2}{*}{ Method } & \multirow[t]{2}{*}{ Unit } & \multicolumn{3}{|c|}{ Clarity level } & \multirow[t]{2}{*}{ Sum } & \multirow[t]{2}{*}{$x^{2}$} & \multirow[t]{2}{*}{$p$} \\
\hline & & $1(\%)$ & $2(\%)$ & $3(\%)$ & & & \\
\hline \multirow[t]{2}{*}{ HRA } & 16-slice & 253 (84.3) & $44(14.7)$ & $3(1.0)$ & 300 & 3.514 & 0.173 \\
\hline & 256-slice & $268(89.3)$ & $29(9.7)$ & $3(1.0)$ & 300 & & \\
\hline \multirow[t]{2}{*}{$\mathrm{HRO}$} & 16-slice & $96(32.0)$ & $168(56.0)$ & $36(12.0)$ & 300 & $10.736^{\mathrm{a}}$ & 0.005 \\
\hline & 256-slice & $114(38.0)$ & $130(43.3)$ & 56 (18.7) & 300 & & \\
\hline \multirow[t]{2}{*}{ SAA } & 16-slice & $135(45.0)$ & $92(30.7)$ & 73 (24.3) & 300 & 3.189 & 0.203 \\
\hline & 256-slice & $143(47.7)$ & $73(24.3)$ & $84(28.0)$ & 300 & & \\
\hline \multirow[t]{2}{*}{ SOA } & 16-slice & $0(0.0)$ & $89(29.7)$ & $211(70.3)$ & 300 & $10.863^{b}$ & 0.004 \\
\hline & 256-slice & $8(2.7)$ & $69(23.0)$ & $223(74.3)$ & 300 & & \\
\hline
\end{tabular}

$C T$ computed tomography, $H R A$ high resolution axial algorithm, $H R O A$ high resolution oblique algorithm, SAA standard axial algorithm, SOA standard oblique algorithm

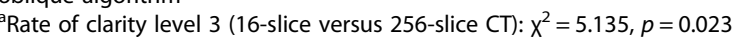

${ }^{b}$ Rate of clarity level 3 plus level 2 (16-slice versus 256-slice CT): $X^{2}=6.208, p=0.013$

$\mathrm{mm}$ before the five-line sign of interlobular fissures can be revealed.

In this study, high-resolution algorithms produced worse results than the standard-resolution algorithm with respect to presenting the five-line sign of the interlobular fissures. We reasoned that in comparison with a standard algorithm, the high-resolution method decreased the image smoothness and increased the sharpness, leading to a clearer microstructural edge in the lung parenchyma. However, this effect also increased image noise that may have overshadowed the density of the pulmonary microstructure.

The purpose of MIP reconstruction is to enhance the brightest pixels, thereby augmenting the contrast $[8,13]$. When applying MIP reconstruction to study diffuse lung lesions, Remy-Jardin et al. [5] used high spatialfrequency reconstruction kernels to facilitate the reconstruction of transverse images in the lung window and noticed reduced intensity of the beaded vessels. This phenomenon was limited to the subpleural area and had

Table 3 Cases of clear or barely clear five-line sign on maximum intensity projection reconstruction in axial or oblique positions using the standard algorithm

\begin{tabular}{|c|c|c|c|c|c|}
\hline \multirow[t]{2}{*}{ Locations } & \multirow[t]{2}{*}{ Cases } & \multicolumn{2}{|l|}{ 16-slice } & \multicolumn{2}{|l|}{ 256-slice } \\
\hline & & Axial (\%) & Oblique (\%) & Axial (\%) & Oblique (\%) \\
\hline Upper right & 50 & $35(70)$ & $15(30)$ & $35(70)$ & $13(26)$ \\
\hline Middle right & 50 & $33(66)$ & $17(34)$ & $40(80)$ & $9(18)$ \\
\hline Lower right & 50 & $0(0)$ & $50(100)$ & $4(8)$ & $45(90)$ \\
\hline Upper left & 50 & $5(10)$ & $45(90)$ & $1(2)$ & $47(94)$ \\
\hline Middle left & 50 & $1(2)$ & 49(98) & $1(2)$ & 48(96) \\
\hline Lower left & 50 & $0(0)$ & $50(100)$ & $0(0)$ & 49(98) \\
\hline$x^{2}$ & & 153.60 & 153.60 & 177.50 & 169.50 \\
\hline$p$ & & $<0.001$ & $<0.001$ & $<0.001$ & $<0.001$ \\
\hline
\end{tabular}

poor imaging quality. This finding easily overcomes the challenge of distinguishing the string-of-beads artificial shadow of MIP-reconstructed microvasculature from the micronodules in diffuse lung lesions. Thus, the control of image noise is a key factor to determine the visibility of the five-line sign (Fig. 3b1-2). Remy-Jardin et al. [5] revealed some pulmonary micronodules that were less than $3 \mathrm{~mm}$ in diameter and had low density and a blurred boundary. MIP reconstruction with $8-\mathrm{mm}$ thickness typically cannot reveal these microstructures, whereas $5-\mathrm{mm}$ thickness is relatively ideal for displaying them. These findings indicate that increasing the MIP thickness may mask low-density structures in the lung, which is consistent with the observation in this study (Fig. 4).

When the standard axial algorithm was used for MIP reconstruction of the five-line sign, the low-clarity rates were $45 \%$ and $48 \%$ for the 16-slice and 256-slice CT scanners, respectively. In contrast, when the standard oblique algorithm was used, the low-clarity rates decreased to $0 \%$ and $2.7 \%$ for the 16 -slice and 256 -slice CT scanners, respectively, because the oblique lines rotated in areas where the oblique fissures were oriented at steep angles, such as the lower segments of the right oblique fissures and all three segments of the left oblique fissures. As a consequence, the oblique fissures and slices formed right angles that are exhibited as clear or barely clear in the most oblique multiplanar reformation images where they otherwise would have had an unclear axial MIP five-line sign, as shown in Fig. 2. In addition, for those areas where the five-line sign was clearly presented by the axial algorithm, the clarity level was not compromised by minor angle changes in the oblique plane. Under the generation of the five-line sign during the MIP reconstruction of interlobular fissures, the fissures and scanning plane must form a suitable angle, which can be easily accomplished by angle adjustment 
Table 4 Included angle between fissures and tangent lines showing a clear or barely clear five-line sign using the standard algorithm

\begin{tabular}{|c|c|c|c|c|c|c|}
\hline \multirow[t]{3}{*}{ Locations } & \multicolumn{3}{|c|}{ 16-slice } & \multicolumn{3}{|c|}{ 256-slice } \\
\hline & Cases & Included angle, degrees & Range, degrees & Cases & Included angle, degrees & Range, degrees \\
\hline & & $M(P 25, P 75)$ & $(\mathrm{P} 2.5, \mathrm{P} 97.5)$ & & $M(P 25, P 75)$ & $(\mathrm{P} 2.5, \mathrm{P} 97.5)$ \\
\hline Right upper & 50 & $-27.00^{\dagger}(-31.15,-20.75)$ & $-54.33 \sim-2.10$ & 48 & $-27.50(-31.00,-19.25)$ & $-40.87 \sim-2.35$ \\
\hline Right middle & 50 & $-0.50(-18.00,17.00)$ & $-43.35 \sim 37.80$ & 49 & $-3.00(-13.00,11.00)$ & $-37.50 \sim 31.00$ \\
\hline Right lower & 50 & $-34.50(-39.00,-31.50)$ & $-45.72 \sim-23.00$ & 49 & $-33.00(-36.00,-28.00)$ & $-44.00 \sim-21.00$ \\
\hline Left upper & 50 & $-31.50(-37.00,-28.00)$ & $-45.72 \sim-16.28$ & 48 & $-33.00(-38.50,-29.00)$ & $-45.55 \sim-20.22$ \\
\hline Left middle & 50 & $-32.00(-36.00,-30.00)$ & $-40.18 \sim-6.42$ & 49 & $-32.00(-35.00,-28.00)$ & $-41.00 \sim-17.25$ \\
\hline Left lower & 50 & $-36.00(-42.00,-31.75)$ & $-46.45 \sim-22.00$ & 49 & $-34.00(-37.50,-31.00)$ & $-46.00 \sim-21.25$ \\
\hline $\mathrm{H}$ & & 113.97 & & & 125.57 & \\
\hline$p^{*}$ & & $<0.001$ & & & $<0.001$ & \\
\hline
\end{tabular}

$\dagger^{\prime \prime}{ }^{\prime \prime}$ Indicates direction only; the absolute values of included angles represent the size

*Differences in angles between fissures and the tangent lines among six locations using the same computed tomography scanner were significant

via multiplanar reformation on the sagittal plane of the workstation. The data in Table 4 show that the median of angles between the tangent lines and the fissures were $-27^{\circ}$ to $-36^{\circ}$ counter clockwise in most cases; the median of the right upper segments was the smallest, in most of which the five-line sign could be revealed in the axial MIP images directly (Table 3), and the median of the left lower segments was the biggest. The minor fissures were oriented at gentle angles with physical curvature (Fig. 2c), and the oblique lines had to be rotated clockwise or counter clockwise to form right angles between the oblique planes and fissures to show the fiveline sign in some cases.

In most areas where the five-line sign could be discerned after adjustment of the oblique angle, the axial MIP images all contained band-like patterns with high density and clear boundaries. In comparison, in a few sites where the five-line sign was invisible after oblique angle adjustment, interlobular fissures in the axial MIP images were all manifested as a stripe-like pattern with low density and blurring boundaries.

This result was corroborated by the $2.7 \%$ of the samples derived from 256-slice CT and the standard oblique algorithm that failed to produce the five-line sign. These findings indicate that the proper density of interlobular fissures and suitable angles between the fissures and the section plane are necessary for the formation of the fiveline sign. This requirement also explains why only the small blood vessels that are oblique to the MIP planes are manifested as strings of beads. Furthermore, the angle between the interlobular fissures and the scanning plane not only influenced the display rate of the five-line sign but also dictated the width of the lines [6]. Finally, in the lower right segment calculated with the highresolution oblique algorithm, and the lower left segment calculated with the standard oblique algorithm, 256-slice CT displayed superior performance to 16-slice CT based on the rate of grading of the five-line sign as clarity level
3. This result may have been observed because imaging of the lower lobes of the lung was strongly affected by the heartbeat; therefore, 256-slice CT generated clearer images because of the faster scanning speed.

To outline the clinical usefulness of the five-line sign, we should consider that normal interlobular fissures feature regular morphology, smooth appearance, and uniform thickness. The MIP-rendered five-line sign has smooth lines and clear gaps and can, therefore, be used as the referencing object for other planar structures in the lung. Certain pulmonary pathological features, such as the pleural tail sign [14-16], represented a form of thickened septa [15]; the five-line sign revealed by MIP reconstruction suggested that it was a relatively smooth and regular plane, and it is difficult to determine using multiplanar or thin-section reconstruction. As we observed that most nodules with the pleural tail sign had an unclear or absent five-line sign, we speculated that the plane was rough and irregular; only a small number of nodules with the tail sign showed a clear five-line sign by MIP rebuilding, but these lines were thicker and denser than those of normal interlobular fissures. Simultaneously, it was noticed in our limited experiments that the tumour was at an advanced stage in most of the cases of lung cancer in which the five-line sign was unclear or absent, and relapse and metastasis usually occurred after resection [17]; whereas a small number of peripheral lung cancers with a clear five-line sign were at an earlier stage and no recurrence and metastases occurred following resection; these patients survived for more than 5 years disease-free [17]. In addition, a doubling time exceeding 800 days was observed in several tumours with a barely clear five-line sign and without experiencing being resected surgically. That implied character of indolent growth of those lung cancers with five-line sign. The cases of lung cancer mentioned involved almost completely solid nodules. Recent clinical studies reported the pleural tail sign or pleural 
indentation as having different clinical effects $[16,18$, 19]; parts of these investigations focused upon pulmonary adenocarcinomas appearing as sub-solid nodules or ground-glass nodules $[18,19]$. Other scholars have applied volume rendering reconstruction technique to predict whether the lung cancer invades the visceral or parietal pleura [20]. Anyhow, the authors of this article attempted to investigate the five-line sign of peripheral lung cancer through MIP reconstruction and to evaluate the effect of the five-line sign on staging and prognosis in peripheral lung cancer, especially when represented as a solid nodule; we consider that it would be worth carrying out further investigation on the basis of the large number of cases accumulated. This is the reason why we explored the five-line sign of the normal interlobular fissures using MIP reconstruction.

This study has limitations. The results were obtained from two different scanners only. Conventional scan parameters were used with half the subjects studied without tube current modulation. We did not obtain data on subjects scanned with both CT scanners (inter-individual and not intra-individual design).

In conclusion, the five-line sign of interlobular fissures can be revealed in MIP reconstructed images derived from different CT scanners. The standard oblique algorithm generated the best reconstruction outcome, whereas the clarity levels were not significantly different between the two CT scanners. The MIP-rendered fiveline sign of normal interlobular fissures can be used as the referencing object for other planar structures in the lung.

\section{Abbreviations}

$C T$ : Computed tomography; MIP: Maximum intensity projection

\section{Acknowledgements}

We deeply express our gratitude to Zhanping He and Yusen Shi for their contributions and assistance to this work.

\section{Funding}

This study has received funding by grants from Scientific Research Funding of Hainan Province (No. ZDYF2017106) of China. One of the authors has significant statistical expertise.

\section{Authors' contributions}

Conception and design, acquisition of data, or analysis and interpretation of data - A.L.Y. and Y.F.Z.; drafting the article or revising it critically for important intellectual content - Q.L., A.L.Y., and J.L.H.; statistical analysis - J.L.H. and Q.L.; final approval of the version to be published - all authors.

\section{Ethics approval and consent to participate}

Institutional Review Boards approval were obtained. Written informed consent was waived by the Institutional Review Boards.

\section{Competing interests}

The authors declare that they have no competing interests.

\section{Author details}

'Department of Radiology, First Affiliated Hospital of Hainan Medical College, 31 Longhua Road, Haikou, Hainan 570102, China. ${ }^{2}$ Department of Pathology, Hainan Medical College, 33 Longhua Road, Haikou, Hainan 570102, China.

${ }^{3}$ Department of Radiology, Haikou People's Hospital, Haikou, Hainan 570208, China. ${ }^{4}$ Department of Ecsomatics, First Affiliated Hospital of Hainan Medical College, 31 Longhua Road, Haikou, Hainan 570102, China.

Received: 5 May 2017 Accepted: 21 August 2017

Published online: 19 October 2017

\section{References}

1. Takahashi K, Thompson B, Stanford W et al (2006) Visualization of normal pulmonary fissures on sagittal multiplanar reconstruction MDCT. AJR Am J Roentgenol 187:389-397

2. Honda O, Johkoh T, Yamamoto S et al (2002) Comparison of quality of multiplanar reconstructions and direct coronal multidetector CT scans of the lung. AJR Am J Roentgenol 179:875-879

3. Remy-Jardin M, Campistron P, Amara A et al (2003) Usefulness of coronal reformations in the diagnostic evaluation of infiltrative lung disease. J Comput Assist Tomogr 27:266-273

4. Sakai M, Murayama S, Gibo M et al (2005) Can maximum intensity projection images with multidetector-row computed tomography help to differentiate between the micronodular distribution of focal and diffuse infiltrative lung diseases? J Comput Assist Tomogr 29:588-591

5. Remy-Jardin M, Remy J, Artaud D, Deschildre F, Duhamel A (1996) Diffuse infiltrative lung disease: clinical value of sliding-thin-slab maximum intensity projection CT scans in the detection of mild micronodular patterns. Radiology 200:333-339

6. Yu A, Li Q, He J, Zhan Y (2014) Conditions and mechanism for the appearance of interlobar fissures as 5 -line signs in axial maximum intensity projection images. J Comput Assist Tomogr 38:578-585.

7. Landis JR, Koch GG (1977) The measurement of observer agreement for categorical data. Biometrics 33:159-174

8. Napel SA (1995) Principles and techniques of 3D spiral CT angiography. In: Jeffrey RB, Fishman EK (eds) Spiral CT. Raven Press, New York, pp 167-196

9. Bhalla M, Naidich DP, McGuinness $G$ et al (1996) Diffuse lung disease: assessment with helical $C T$-preliminary observations of the role of maximum and minimum intensity projection images. Radiology 200:341-47

10. Lee HY, Goo JM, Lee HJ et al (2009) Usefulness of concurrent reading using thin-section and thick-section CT images in subcentimetre solitary pulmonary nodules. Clin Radiol 64:127-132

11. National Lung Screening Trial Research Team (2011) The national lung screening trial: overview and study design. Radiology 258:243-253

12. van Klaveren RJ, Oudkerk M, Prokop M et al (2009) Management of lung nodules detected by volume CT scanning. N Engl J Med 361:2221-2229

13. Napel S, Rubin GD, Jeffrey RB Jr (1993) STS-MIP: a new reconstruction technique for $C T$ of the chest. J Comput Assist Tomogr 17:832-838

14. Hill CA (1982) "Tail" signs associated with pulmonary lesions: critical reappraisal. AJR Am J Roentgenol 139:311-316

15. Webb WR (1978) The pleural tail sign. Radiology 127:309-313

16. Hsu JS, Han IT, Tsai TH et al (2016) Pleural tags on CT scans to predict visceral pleural invasion of non-small cell lung cancer that does not abut the pleura. Radiology 279:590-596

17. Yu A, Li Q (2010) Role of the five line sign on peripheral lung cancer in diagnosis and prognosis. Paper presented at the 26th international congress of radiology (ICR 2010), Shanghai, China, 9-10 April 2010

18. Zhang Y, Shen Y, Qiang JW et al (2016) HRCT features distinguishing pre-invasive from invasive pulmonary adenocarcinomas appearing as ground-glass nodules. Eur Radiol 26:2921-2928

19. Zhao LL, Xie HK, Zhang LP et al (2016) Visceral pleural invasion in lung adenocarcinoma $\leq 3 \mathrm{~cm}$ with ground-glass opacity: a clinical, pathological and radiological study. J Thorac Dis 8:1788-1797

20. Ebara K, Takashima S, Jiang B et al (2015) Pleural invasion by peripheral lung cancer: prediction with three-dimensional CT. Acad Radiol 22:310-319

\section{Publisher's Note}

Springer Nature remains neutral with regard to jurisdictional claims in published maps and institutional affiliations. 